Communication, technologies et développement

5 | 2018

Le numérique et le développement des Suds

\title{
Critique de la gouvernance numérique
}

Criticism of digital governance

Crítica de la gobernanza digital

\section{Bonaventure Mve Ondo}

\section{(2) OpenEdition}

Journals

Édition électronique

URL : http://journals.openedition.org/ctd/301

DOI : $10.4000 /$ ctd. 301

ISSN : 2491-1437

Éditeur

Chaire Unesco Pratiques émergentes en technologies et communication pour le développement

Référence électronique

Bonaventure Mve Ondo, "Critique de la gouvernance numérique », Communication, technologies et développement [En ligne], 5 | 2018, mis en ligne le 02 janvier 2018, consulté le 21 avril 2019. URL: http://journals.openedition.org/ctd/301; DOI : 10.4000/ctd.301

Ce document a été généré automatiquement le 21 avril 2019

Communication, technologies et développement 


\title{
Critique de la gouvernance numérique
}

\author{
Criticism of digital governance \\ Crítica de la gobernanza digital
}

Bonaventure Mve Ondo

1 Cartes d'identité et passeports biométriques, cartes d'électeurs ou d'étudiants biométriques, empreintes numériques, systèmes d'informations géographiques, gestion informatisée des universités et même des élections, le numérique a envahi toutes les sphères des sociétés dans leurs activités de production, de gestion, de contrôle et de diffusion de l'information. En Afrique, la plupart des États tentent de mettre en place ce type d'instruments pour assurer une gestion moderne et plus apaisée tant des élections, des universités, que plus généralement de la société (e-administration). Il est vrai que, dans ces pays, la gouvernance, et plus particulièrement la gouvernance politique, n'a cessé de poser problème depuis les indépendances, tant elle est l'objet de contestations, et par conséquent de crises et de violences de toutes sortes.

2 Voilà en effet que depuis bien longtemps l'Afrique a mal à sa gouvernance ! Et les maux sont légion : abus de pouvoir, banalisation de la corruption, trafics d'informations en tous genres $^{1}$, absence de performance, détournements des biens, méconnaissance des droits élémentaires des individus, ignorance des notions d'intérêt général, de service public et de l'état de droit... Et c'est pour tenter de sortir de cette insécurité administrative générale que les États africains ont commencé à avoir recours, depuis une dizaine d'années, à la gouvernance par les systèmes d'information ou à la gouvernance numérique. Cette dernière est même apparue comme une solution majeure. En matière électorale, elle est souvent présentée aujourd'hui comme l'outil de gestion et de sécurisation de la démocratisation de la vie politique, capable d'assurer la transparence et de garantir l'impartialité des scrutins.

3 Parce qu'il vise à renforcer tout à la fois la confidentialité des informations et la sécurité des communications, ce nouveau mode de gouvernance entend concilier deux exigences contraires, la sécurité et la liberté, et assurer l'exigence démocratique par un système 
technologique. Mais ce souci vertueux ne le met pas à l'abri du soupçon démocraticide en ce qu'il peut apparaître, à certains, comme un cartel organisé d'intérêts politiques, économiques et financiers, nationaux ou étrangers ${ }^{2}$ qui prend une part prépondérante dans la gestion administrative et politique du pays.

Dans tous les cas, nombre de questions demeurent: qu'est-ce que la gouvernance numérique? Quels sont ses usages et ses logiques? Dans quelle mesure constitue-t-elle, dans les processus de gestion électorale, une réponse possible devant la mal-gouvernance, les désordres invoqués, les dérives contestataires et la violence? En ce qu'elle vise à assurer la traçabilité des personnes, n'est-elle pas une menace du droit des individus à la vie privée, et donc des libertés ? Enfin, à quelles conditions ce mode de gouvernance peutil permettre de concilier sécurité et libertés et assurer l'organisation d'une vie démocratique véritable?

\section{Ce qu'est la gouvernance}

D’une manière générale, on définit la gouvernance comme la manière dont les différents acteurs, étatiques et non étatiques, prennent des décisions publiques et gèrent les ressources économiques et sociales au service du développement d'un pays. Et celle-ci comporte plusieurs dimensions qui sont, entre autres : sûreté et sécurité, état de droit, participation politique, droits de l'homme, gestion du secteur public, environnement des affaires et inclusion sociale. Selon Jean-François Chantaraud, «la gouvernance est l'ensemble des règles et méthodes organisant la réflexion, la décision et le contrôle de l'application des décisions au sein d'un corps social »'. Mais si donc la gouvernance est entendue comme l'art de bien gouverner, il n'en reste pas moins qu'elle porte de plus en plus sur des domaines spécifiques et divers comme l'enseignement supérieur ${ }^{4}$, les élections ${ }^{5}$, ou même les systèmes d'information ${ }^{6}$, en même temps qu'elle devient de plus en plus technique. Et c'est pour bien faire ressortir cette dernière dimension que Michel Foucault a effectué le déplacement de la théorisation de l'État à son analyse sous l'angle de ses pratiques, c'est-à-dire de sa gouvernementalité définie comme un mode spécifique d'exercice du pouvoir. Celui-ci n'est plus une substance ou une essence, mais un lieu qui organise les relations de pouvoir. L'État, ce sont des faits, des transactions, l'effet mobile d'un régime pour assurer la gouvernabilité ${ }^{7}$ de la société. Considérant que l'État n'est plus un absolu, Michel Foucault invite donc à investir les rationalités implicites de tout pouvoir, autrement dit de la gouvernance :

«Il me semble en effet qu'à travers la crise économique actuelle et des grandes oppositions et conflits qui se dessinent entre nations riches et pauvres (entre pays industrialisés et non industrialisés), on peut voir la naissance d'une crise de gouvernement. Par gouvernement, j'entends l'ensemble des institutions et pratiques à travers lesquelles on guide les hommes depuis l'administration jusqu'à l'éducation. C'est cet ensemble de procédures, de techniques, de méthodes qui garantissent le guidage des hommes les uns par les autres qui me semble aujourd'hui en crise (...). Nous sommes peut-être au début d'une grande crise de réévaluation du problème de gouvernement $»^{8}$.

6 Une telle analyse du pouvoir et de ses mécanismes peut aider à les sécuriser et donc s'adjoindre des outils tels que le numérique. C'est dans ce sens que l'on peut parler aujourd'hui de la gouvernance numérique. 


\section{De la gouvernance numérique}

7 D'une manière spécifique, la gouvernance numérique est une technique qui recourt à l'analyse de données, à leurs mises en relation pour sortir du système traditionnel de gestion des institutions. Pour ce qui concerne la gouvernance numérique de l'enseignement supérieur dans un contexte mondial globalisé et fortement concurrentiel, il est de plus en plus admis, même dans les pays du Sud, que les universités doivent s'appuyer sur toutes les ressources à leur disposition pour mener à bien leurs missions. La conséquence de tout ceci, pour qu'elles demeurent compétitives et attractives, c'est qu'elles doivent non seulement améliorer toutes leurs activités, telles que l'enseignement, la recherche, la diffusion du savoir et l'insertion professionnelle des diplômés, mais aussi faire évoluer leurs systèmes d'information et leurs ressources pour faciliter leur pilotage, leur gestion stratégique et la prise de décision de leurs responsables. Mais encore faudrat-il s'assurer de la sécurisation de leurs données et de la fiabilité de ces dernières !

8 L'usage des systèmes d'information peut viser deux fins distinctes, qui constituent des aspects importants du débat sécurité / libertés en ce qu'elles permettent d'une part de vérifier l'identité du sujet par rapport aux données dont on dispose sur lui, et d'autre part, d'identifier le sujet par rapport à une base de données afin de le reconnaître. Dans le premier cas, la constitution d'une telle base n'est pas nécessaire, dans le second, elle l'est. Mais dans tous les cas, ce mode de gouvernance, même s'il a des avantages, suscite des appréhensions en raison des risques qui lui sont inhérents. En effet, parce que les informations sont mises en place par des hommes, il n'y aura jamais de risque zéro pour la falsification des informations ou leur utilisation à des fins frauduleuses. Réapparaît ici le débat aujourd'hui récurrent entre les tenants de la sécurité et ceux de la défense des libertés.

\section{Le numérique liberticide}

D'une manière générale, l'arrivée des systèmes d'information et leur interconnexion sont considérées comme une avancée, que ce soit pour gérer les identités, les étudiants et plus encore les élections. S'agissant plus particulièrement de ces dernières, si au départ l'objectif était de vérifier l'identité d'une personne sur une liste électorale en permettant d'éviter les doublons et autres fraudes, il s'agit désormais de certifier son identité, c'est-àdire de la sécuriser, de pouvoir faire des distinctions entre les «nationaux» et les " étrangers ", ou parfois même, comme on peut l'imaginer, entre les «bons » électeurs et les autres. De cette manière, ce qui constituait dans le mode classique d'identification des individus, à savoir la reconnaissance par le face-à-face, par la socialisation, par le vivre ensemble, est ainsi écarté, tout comme d'ailleurs les fameux " papiers d'identité ». Ce qui compte désormais, c'est ce que dit la machine. Ainsi, on tente de transformer ou de réduire un problème social et politique (l'impossible démocratie électorale) à un problème purement technique. On voit, d'ailleurs avec quelle rapidité, en Afrique, les gouvernements comme les oppositions se sont accordés sur la nécessité de ce nouveau mode de gouvernance. Or, celui-ci n'en est pas moins liberticide ${ }^{9}$.

En effet, le système d'informations biométriques, tel qu'il est mis en place, transforme peu à peu la société actuelle écartelée entre le souci démocratique et les pratiques dictatoriales en une "société de la surveillance ", en une société de contrôle qui repose 
sur un fichage généralisé. En permettant de substituer les papiers d'identité classiques à une carte d'identité numérique qui évite certes toute usurpation et falsification d'identité, en contrôlant toutes les informations, on aboutit à mettre en place un pouvoir omniscient de l'État qui, pour ses besoins ou pour ceux qu'il sert, peut violer le droit à la vie privée et donc met à mal les libertés individuelles à travers le principe de traçabilité. En renforçant ainsi le pouvoir de l'État suivant l'impératif de sécurité, s'établit une sorte de coup d'État permanent qui conforte le pouvoir de l'administration contre l'individu et la société, qui empêcherait à terme toute alternance politique et renforcerait les dictatures. La nouvelle gouvernance numérique, au lieu de renforcer la démocratie, viendrait conforter la fameuse théorie du complot des gouvernants contre la démocratie à travers une sorte de colonisation de la vie privée et de l'information ${ }^{10}$.

11 Ainsi, comme le dit l'adage, qui détient l'information, détient aussi le pouvoir. Si donc le soupçon de la tentation totalitaire n'est jamais levé en ce qui concerne ce nouveau mode de gouvernance électorale, plusieurs États soulignent qu'il n'est qu'un outil au service de la démocratie et des organismes qui la gèrent comme les commissions nationales indépendantes ou les cours constitutionnelles. Or, ces deux dernières institutions ne sont ni tout à fait indépendantes, ni pas toujours consensuelles dans leur fonctionnement. Dans la plupart des pays, les membres de ces commissions sont choisis essentiellement selon le critère politique. La commission devient donc très vite le reflet des crises politiques nationales et non un arbitre véritablement indépendant. Du coup, son pouvoir de décision est très faible et son inféodation quasi naturelle avec l'administration (ministère de l'Intérieur) ou avec le parti au pouvoir. En plus, elle ne dispose le plus souvent pas de compétences techniques qui lui permettraient d'assurer une analyse critique de ses outils ou des dispositifs et autres systèmes d'information et de communication mis à sa disposition par l'État. En ce qui concerne les cours constitutionnelles, elles ont certes, comme les précédentes, un fondement juridique, mais le mode de désignation de leurs membres selon la logique majoritaire en fait, comme le note si bien El Hadj Mbodj, des structures d'enregistrement, d'approbation et de ratification des décisions prises ailleurs ${ }^{11}$. Elles sont donc ainsi «inaptes à garantir la sincérité du scrutin ${ }^{12} »$.

Pour bien comprendre comment on en est arrivé là, il faut partir du fait que si les élections libres et démocratiques en Afrique sont devenues depuis les années 1990 un critère de base de la démocratie, il n'en a pas toujours été ainsi. Dès l'avènement des indépendances politiques, ce sont des régimes autoritaires qui ont été mis en place, c'està-dire des régimes qui prétendaient gouverner la quasi-totalité des aspects de la vie des citoyens et qui ne toléraient aucune opposition sérieuse à leurs projets. Au nom, souvent, d'une nécessité de construire des Nations balbutiantes, ou de réaliser le développement économique et social, les pouvoirs en place se montraient peu respectueux des libertés civiles et du droit à l'objection. L'Afrique a vécu ainsi des élections sans choix ou des simulacres d'élections qui étaient souvent organisées, avec des résultats connus d'avance et des scores plébiscitaires pour les gouvernants. Dans les années 90 , avec la chute du mur de Berlin et le fameux discours de La Baule, les premières alternances politiques ont eu lieu qui ont abouti à la nécessité de mettre en place de nouvelles structures chargées de l'organisation des élections. Il s'agissait donc de sortir de la gestion habituelle de ce moment important de la vie démocratique par les ministères de l'Intérieur qui n'inspiraient plus confiance pour une organisation transparente et équitable de la compétition électorale. L'ouverture démocratique des années 90 a donc été caractérisée 
par la mise en place, un peu partout, d'organes censés gérer, en toute indépendance, les processus électoraux. De rudes batailles ont opposé à ce sujet les tenants du pouvoir et les opposants, la configuration donnée à ces commissions électorales passant pour être révélatrice de la réelle volonté des autorités à promouvoir des élections libres, sincères et démocratiques. L'apprentissage de la pratique électorale a donc réconcilié l'Afrique avec la démocratie pluraliste. Ces nouvelles structures censées être les nouvelles vigies de la démocratie ont nourri beaucoup d'espoirs. Mais plus d'une trentaine d'années après les premières expériences d'organisation d'élections libres et compétitives, elles sont devenues elles-mêmes très contestées et les résultats des élections sont toujours sujets à caution. Ces dernières sont même devenues, dans la plupart des pays africains francophones, conflictogènes, que ce soit lors de leurs préparations, de leur déroulement ou même de leur proclamation.

On le voit, le calcul utilitariste et la logique consensuelle qui ont été à l'origine de ces structures ont été vite débordés par les passions politiques. Et tant que le moindre soupçon de partialité demeure sur leur mode de gouvernance, ces institutions n'ont pas permis d'imposer la transparence et la paix. Et s'il en était de même pour la gouvernance numérique? Et si, à l'image du scandale Volkswagen, il y avait, au cœur des systèmes d'information électorales biométriques, un logiciel « truqueur » qui favoriserait le groupe politique qui est en lien, au-delà des commissions nationales indépendantes, avec l'entreprise qui a implémenté ces systèmes, une machine « venue de l'étranger » et qui ferait toujours gagner le candidat du complexe « international et politico-industriel » ? Il $\mathrm{y}$ a là un pas que nous ne franchirons pas, mais que franchissent allègrement certains médias nationaux et internationaux ${ }^{13}$. Il n'empêche qu'il importe plus que jamais de sortir de la logique du soupçon, de réfléchir sur les pratiques et de déterminer les conditions sine qua non d'une gouvernance numérique qui allie sécurité et libertés, démocratie formelle et volonté populaire.

\section{Pour une gestion « démocratique » de la gouvernance numérique}

Dans la gestion des élections, ni le tout technologique, ni le poids des commissions nationales électorales indépendantes n'ont permis d'instaurer la confiance et d'éliminer tous les soupçons de collusion. Peut-être qu'à l'exemple de ce qui s'est passé, pour la première fois, lors des élections au Sénégal en 2000 et qui est en train de se mettre en place dans l'enseignement supérieur au Mali, il est possible de dégager les principes communs qui allient système d'information et bonne gouvernance et qui peuvent aider à faire évoluer de manière positive et durable les pratiques.

\section{L'exemple du Sénégal en 2000}

Nous l'avons déjà dit plus haut, l'impasse actuelle du processus de démocratisation en Afrique subsaharienne provient de la contestation des élections. Si, depuis les années 1990, des avancées significatives ont été réalisées en matière de liberté d'expression et des droits de l'homme, il est généralement admis que l'organisation des élections libres et transparentes reste l'un des principaux points de blocage de l'évolution démocratique en Afrique et qu'elle est à l'origine de la plupart des crises politiques qui secouent le continent. C'est dire que la bonne gouvernance des élections représente le défi majeur 
pour la transition vers le pluralisme démocratique et constitue donc l'une des conditions incontournables de sa stabilité politique et sociale.

Par ailleurs, il est avéré que l'introduction des TIC dans le processus électoral, sans pour autant être à elle seule capable de régler la complexité de la question des élections, peut contribuer à améliorer la transparence et la démocratie comme l'a démontré le Sénégal en l'an 2000.

En effet, le pays a mené une expérience intéressante d'utilisation des TIC dans l'organisation et la gestion des élections cette année-là. Tout d'abord, le fichier électoral, qui avait été informatisé en 1977, avait été mis en ligne. Ensuite, des terminaux informatiques avaient même été installés dans des lieux publics et dans les sièges de certains partis politiques. Tout cela a permis d'assurer à tout un chacun un accès égal et transparent aux fichiers, aussi bien aux partis politiques qu'à l'ensemble de la population, et donc de s'inscrire. À tel point que le jour où le système fut mis en ligne sur internet, plus de 21000 personnes y accédèrent. Pour Lamine Cissé, alors ministre de l'Intérieur, chargé d'organiser les élections, « le recours aux nouvelles technologies de l'information et de la communication a permis de clore le débat sur la fiabilité du fichier, mais surtout, de banaliser l'accès à ce fichier. [...] Cela donne à ces électeurs un sentiment de puissance (responsabilité citoyenne), de maîtrise d'informations concernant le processus électoral qui les concerne et pour lequel ils constituent des acteurs majeurs, bien que moins visibles et moins audibles que les autres groupes d'acteurs structurés comme les partis politiques et les organes de contrôle ${ }^{14} »$.

Ainsi les TIC ont permis un contrôle populaire du processus de décompte des voix, évitant ainsi les tripatouillages. Selon Christian Valantin, si les trois types de contrôle du processus électoral, à savoir le contrôle de régularité assuré par les commissions de recensement, l'office national des élections et la cour d'appel, le contrôle juridictionnel par le Conseil constitutionnel et le contrôle des observateurs internationaux, ont bien eu lieu, «il faut y ajouter un autre type de contrôle, celui des médias et des nouvelles technologies de l'information et de la communication (NTIC), en particulier des mobiles. C'est la première fois au Sénégal que les médias (radio et presse) ont occupé une telle place dans une élection, que l'information est remontée et a été diffusée presque en temps réel grâce au téléphone mobile. L'élection était ainsi placée sous le contrôle de la société civile ${ }^{15}$ ».

19 Avec de telles pratiques s'est ainsi constituée et renforcée au Sénégal une nouvelle tradition électorale. Ce qui a fait dire à Barack Obama, le Président des États-Unis d'Amérique, qu' en ce XXI $^{\mathrm{e}}$ siècle, des institutions capables, fiables et transparentes sont la clé du succès [...]. L'Afrique n'a pas besoin d'hommes forts, mais de fortes institutions ${ }^{16}$ ».

\section{L'exemple de la gouvernance numérique des universités}

Que ce soit au Cameroun, au Gabon, au Mali, des expériences d'un mode de gestion des universités ou du moins de quelques volets de celle-ci ont été tentées. Á l'Université de Yaoundé II par exemple, il s'est agi, en son temps, de sécuriser l'inscription et les séances d'examen pour vérifier la présence réelle des candidats. Á Libreville, une expérience de gestion informatique avait été mise en place en 1991, dénommée GESTUX. L'objectif était ici de sécuriser ici à la fois la gestion financière et la gestion et le suivi des étudiants. Mais l'expérience la plus engagée est celle qui est actuellement mise en place au Mali par un 
consortium international et dirigé par une entreprise néerlandaise, le CINOP, avec des experts de l'AUA, des universités du Gabon, du Mali et de France, mais aussi avec l'appui d'une entreprise d'informatique française, Cocktail. Il s'agit plus concrètement d'assurer l'inscription ainsi que le suivi administratif et pédagogique des 80000 étudiants des grandes écoles et des universités. Au-delà de l'outil technique qu'est le progiciel Cocktail, ce qui est remarquable dans ce projet, c'est la création d'un fichier crédible étudiant qui évite les doublons et qui réduit toutes les formes de résistances ${ }^{17}$. Il faut rappeler que jusqu'ici l'inscription dans les institutions d'enseignement supérieur du Mali était un acte courageux, l'impétrant subissant toutes formes de pressions, d'escroquerie et de violences qui s'installaient le plus souvent en amont de l'administration universitaire.

21 Ainsi, avec une gouvernance numérique qui assure la transparence des processus, il est possible de réduire les poches d'incertitude et de restaurer la vie démocratique et la confiance. Cela suppose en réalité de recréer le fameux principe de la discussion élaboré par Jürgen Habermas ${ }^{18}$ et amélioré par Nelson Mandela ${ }^{19}$, à travers une concertation permanente et d'assurer le libre contrôle de l'ensemble de la chaîne d'information par les acteurs eux-mêmes. Le premier principe peut être adossé à une charte de la démocratie et des libertés à laquelle adhéreraient toutes les parties prenantes (partis politiques, administration, société civile, mais aussi ONG, experts scientifiques et techniques), mais aussi les citoyens. Le second concerne leur participation, dans un esprit de collégialité, à l'ensemble du processus, de la conception, à l'implémentation, à la révision du fichier et à la proclamation des résultats ${ }^{20}$, avec, si possible, d'autres outils de contrôle indépendants et facilement lisibles. Cela suppose enfin que les décomptes soient publics et diffusés à travers les médias publics ${ }^{21}$.

En effet, c'est par la conformité des résultats établis publiquement dans un bureau de vote et ceux diffusés par les commissions nationales des élections et la Cour constitutionnelle avec ceux affichés par les observateurs indépendants et les médias que se vérifiera la fiabilité de tout le système. La leçon générale, c'est qu'il n'y aura de transparence électorale que si les processus d'identification, de vérification et de diffusion des résultats sont indépendants des autorités politiques en place. En effet, dans le flou actuel, ce n'est pas parce qu'un individu possède des papiers d'identité avérés que son inscription sur les listes électorales est définitive. Encore faut-il que là aussi la reconnaissance sociale joue et permette de mettre hors-jeu tout transport de personnes et toute tentative de fraude.

\section{Conclusion}

Ainsi, même si la technologie assure l'unité du processus et la sécurité des informations, elle n'est pas fondamentale dans la construction de la confiance sociale. Ce qui est important, c'est l'éthique de la discussion et de la transparence. L'on voit donc alors qu'une gestion " démocratique » de la gouvernance numérique suppose deux préalables, à savoir: assurer l'indépendance véritable des acteurs qui y participent (entreprises, partis politiques, administrations, observateurs extérieurs, etc.) et permettre à tous les citoyens l'accès aux informations et leur diffusion librement et en temps réel.

Dans tous les cas, aucun mode de gouvernance, fût-il numérique, ne peut fonctionner de façon durable et saine sans un minimum de sécurité, sans un minimum de normes admises par tous, sans un minimum de confiance entre les acteurs. L'erreur des élites 
politiques africaines est de ne pas l'avoir compris plus tôt. Il en résulte une situation de désenchantement et d'anomie, une situation de délitement de l'État qui est au seul service de ceux qui le dirigent. C'est le propre de toute gouvernance dictatoriale et autoritaire. Plus que jamais, le défi de toute gouvernance crédible exige que l'on sorte des luttes idéologiques, voire ethniques, pour se concentrer sur la nécessaire restauration de la confiance autour et avec des citoyens plus responsables et plus déterminés. Car aucune politique démocratique véritable n'est pensable, ni même possible sans un minimum de principes, qui sont ceux du droit et de l'éthique.

Ainsi ceux qui croient qu'on peut réduire la question sociale et politique de la vie démocratique à un simple problème technique se fourvoient. Mieux, plus que jamais, il ne saurait y avoir de développement valable et durable sans une participation sociale véritable, sans une appropriation claire et sans une connaissance fine de celle-ci par tous les acteurs. Mais si les conditions de la confiance sociale dans un système de gestion démocratique peuvent être connues et explicitées, rien ne sous dit qu'elles sont suffisantes, voire simplement rationnelles dans leur totalité. Et si au-delà de ces conditions, la condition première de toute démocratie n'était pas simplement le gouvernement du peuple par le peuple et pour le peuple, ni même les techniques et les outils, mais d'abord la liberté de penser et l'exigence d'apprendre à penser autrement, alors la démocratie ne serait jamais achevée, mais en perpétuelle construction tout en reposant sur des principes universels et en vue d'éliminer toutes les sources de malentendus, de dissentiments, de conflits et de ressentiments.

\section{BIBLIOGRAPHIE}

Atlan Monique et Droit Roger-Paul, Humain. Une enquête philosophique sur ces révolutions qui changent nos vies, Flammarion, 2012.

Biagini Cédric, L'Emprise numérique. Comment Internet et les nouvelles technologies ont colonisé nos vies, L'Echappée, 2012.

Cardon Dominique, La Démocratie internet. Promesses et limites, Le Seuil, coll. « La République des idées ", Paris, 2010.

Chantaraud Jean-François, " Gouvernance », dans Chantaraud Jean-François, Glossaire, Observatoire du dialogue et de l'intelligence sociale, Paris, 2010, disponible sur http://www.portailhumanitaire.org/directory/listing/resodi.

Denef Jean-François, Mvé-Ondo Bonaventure (dir.), Introduction à la gouvernance des universités. Guide de gouvernance et d'évaluation à l'usage des recteurs et présidents d'universités ou d'institutions d'enseignement supérieur, Éditions des archives contemporaines / Agence universitaire de la francophonie / Institut de la francophonie pour la gouvernance universitaire, Paris, 2015.

Du Bois de Gaudusson Jean, «Les élections à l'épreuve de l'Afrique », Les Nouveaux Cahiers du Conseil constitutionnel, no 13 (La Sincérité du scrutin), 2003, disponible sur http://www.conseilconstitutionnel.fr. 
Dugain Marc et Labbé Christophe, L'homme nu. La dictature invisible du numérique, Lafond, Plon, 2016.

Foucault Michel, Les Mots et les Choses. Une archéologie des sciences humaines, Gallimard, coll. «Bibliothèque des sciences humaines », Paris, 1966.

Foucault Michel, Dits et Écrits. Volume III : 1976-1979, édition établie sous la direction de Defert Daniel et Ewald François, avec la collaboration de Lagrange Jacques, Gallimard, coll.

«Bibliothèque des sciences humaines », Paris, 1994.

Foucault Michel, Sécurité, territoire, population. Cours au Collège de France. 1977-1978, Gallimard / Le Seuil, coll. « Hautes études », Paris, 2004.

Habermas Jürgen, De l'éthique de la discussion, traduit de l'allemand par Hunyadi Mark, Éditions du

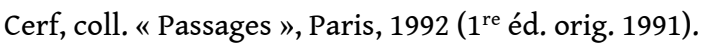

Godart Elsa, Je selfie donc je suis, Albin Michel, Paris, 2016.

Mandela Nelson, Un long chemin vers la liberté, Livre de poche, Fayard, 1995.

Mbodj El Hadj, « Faut-il avoir peur de l'indépendance des institutions électorales en Afrique ? ", Afrilex, 2009, disponible sur http://afrilex.u-bordeaux4.fr.

Morozov Evgeny, Pour tout résoudre, cliquez ici. L'aberration du solutionnisme technologique, FYP éditions, 2014.

Mvé-Ondo Bonaventure, « Le Gabon en danger de mort », Septembre 2016, in http://theconversation.com/le-gabon-en-danger-de-mort-65061.

Ntoutoume Loïc, «Libertés individuelles : les Gabonais espionnés par FinFisher ? », Gabon Review, 2015, disponible sur http://gabonreview.com.

Ossama François, « Défis pour l'approfondissement du processus démocratique en Afrique subsaharienne ", Netsuds, $n^{\circ} 1$ (Les Fractures numériques Nord/Sud en question), 2003.

Sadin Eric, La vie algorithmique. Critique de la raison numérique ; l'Echappée ; 2015.

Thales, « Gestion d'identité sécurisée. La biométrie au cœur des solutions globales », bro- chure, s.d., disponible sur http://www.thalesgroup.com/en

Valantin Christian, «L'élection présidentielle de 2000 au Sénégal », dans Actes de la troi- sième réunion préparatoire au symposium de Bamako : les élections (Paris, 25-27 avril 2000), Organisation internationale de la francophonie, Paris, s.d., disponible sur http:// www.francophonie.org.

Vial Stéphane, L'être et l'écran. Comment le numérique change la perception; PUF ; 2013. Wolton Dominique, L'Autre mondialisation; Flammarion, 2003.

Wormser Gérard, «Banco! L'espace public à l'heure du numérique », Sens public, 2010, disponible sur http://sens-public.org.

\section{NOTES}

1. Comme par exemple ceux des actes de naissance et autres jugements supplétifs et ceux des notes d'examens dans les systèmes éducatifs.

2. Cf. G. Wormser, 2010.

3. Chantaraud, 2010. 
4. C'est dans ce cadre que nous avons appuyé en son temps au sein de l'AUF la création de l'Institut panafricain de gouvernance universitaire (IPAGU) en 2010, qui est devenu par la suite l'Institut de la francophonie pour la gouvernance universitaire (IFGU), installé dans le campus de l'université de Yaoundé II, au Cameroun. Un maître ouvrage a d'ailleurs été publié dans ce cadre que nous avons dirigé avec J.-F. Denef: Introduction à la gouvernance des universités. Guide de gouvernance et d'évaluation à l'usage des recteurs et présidents d'université ou d'institutions d'enseignement supérieur, (Denef, Mve-Ondo [dir.], 2014).

5. Pour l'un des grands groupes européens de réalisation de solutions globales qu'est Thales et dont le maître mot est la sécurité, l'objectif est de «simplifier les relations entre l'État et les élections en facilitant, par exemple, l'accès aux élections, aux services sociaux ou à l'emploi » (Thales, s.d.)

6. Il faut bien sûr distinguer la gouvernance de l'Internet de la gouvernance numérique ou par les TIC. La première est un dispositif international qui vise à gérer et à partager les responsabilités entre les principaux acteurs de l'Internet. La seconde utilise le numérique comme un outil d'amélioration de la gouvernance. Il s'agit de dispositifs ou d'outils d'aide à l'analyse et donc à la décision.

7. Foucault, 1966, 1994 et 2004.

8. Foucault, 1994, pp. 93-94.

9. Pour Dominique Cardon, le développement de l'internet «bouleverse notre conception et notre pratique de la démocratie » (Cardon, 2010, p. 7).

10. On ne peut que renvoyer ici au hold-up électoral réalisé en septembre 2016 au Gabon qui a montré comment, en une nuit, le pouvoir en place a transformé les électeurs d'une région qui aurait voté à plus de $99 \%$ en un facteur d'ajustement des résultats et surtout n'a jamais voulu confronter les résultats détenus par les citoyens et les observateurs avec ceux détricotés par leurs services.

11. Mbodj, 2009.

12. Du Bois de Gaudusson, 2003, p. 101.

13. Voir le très sérieux article de Loïc Ntoutoume (Ntoutoume, 2015).

14. Cité dans Ossama, 2003, p. 100.

15. Valantin, s.d.

16. Discours d'Accra, Ghana, 12 juillet 2009.

17. Cf. le site http://www.campusmali.ml

18. "Selon l'éthique de la discussion, une norme ne peut prétendre à la validité que si toutes les personnes qui peuvent être concernées sont d'accord (ou pourraient l'être) en tant que participant à une discussion pratique sur la validité de cette norme ", (Habermas, 1992 [1 $1^{\text {re }}$ éd. Orig. 1991], p. 76).

19. Nelson Mandela a montré que c'est par le dialogue, un dialogue qui repose sur les principes essentiels de justice, d'égalité et de liberté que la réconciliation et la vie démocratique sont possibles. Il s'agit du «dialogue sans exclusive » selon la célèbre formule «Un homme, une voix ".

20. C'est le principe de la co-construction, de la co-gestion et de la co-responsabilité.

21. Un exemple de " corrections », notamment en ce qui concerne la population électorale et les résultats, c'est notamment pour l'élection présidentielle de 2016 au Gabon où la population de la région du Haut-Ogooué a été multipliée en une nuit (celle du 30 au 31 août) par 4 par la célèbre Commission nationale des élections pour pouvoir délivrer un résultat conforme aux attentes du pouvoir en place, malgré les injonctions des observateurs étrangers. 


\section{RÉSUMÉS}

Le nouveau contexte géopolitique issu du 11 septembre 2001 a mis en avant le recours à la biométrie dans les aéroports comme outil de sûreté, de sécurité et de contrôle pour faciliter l'identification et l'authentification massive des individus. Ce dispositif est désormais étendu au processus électoral dans l'ensemble des pays du monde.

En Afrique, beaucoup de pays l'ont adopté pour tenter de crédibiliser les élections, des contextes endogènes liés à l'opacité du mode de fonctionnement de ces institutions. C'est pourquoi la gouvernance numérique, si elle veut redonner la confiance et assurer la transparence, doit s'appuyer sur l'implication réelle et libre des acteurs sociaux eux-mêmes.

The new geopolitical context of September 11 has highlighted the use of biometrics in airports as a tool for safety, security and control for the massive identification and authentication of individuals. This system has now been extended to electoral process worldwide.

In Africa, several countries have adopted this system as a means to give credibility to elections even though it is neither perfect nor unquestionable with regard to endogenous contexts related to the opacity of the functioning mode of these institutions. Accordingly; digital governance should rely upon a real and free involvement of social actors themselves if it aims to restore trust and ensure transparency.

A raíz del 11 de septiembre 2001, el nuevo panorama geopolítico ha implantado la biometría en los controles aeroportuarios como medida de protección y herramienta de seguridad para facilitar la identificación y autentificación masiva de flujos migratorios, tanto que, dicho dispositivo se ha extendido en la actualidad, a los procesos electorales en el conjunto de países mundiales.

En África, muchos países lo han adoptado para dar credibilidad y validez a sus elecciones, aunque, la biometría, como sistema de gestión y generador de bases de datos, no es infalible. Incluso, es muy discutible debido a contextos endógenos en los que persiste la opacidad, un escollo que dificulta el modo de funcionamiento de determinadas instituciones africanas. Por eso, si pretende devolver la confianza y asegurar la transparencia, la gobernanza digital, ha de contar, de forma real y libre, con la labor de los actores sociales.

\section{INDEX}

Palabras claves : gobernabilidad, elecciones, digital, Africa

Keywords : governance, elections, digital, Africa

Mots-clés : gouvernance, élections, numérique, Afrique

\section{AUTEUR}

\section{BONAVENTURE MVE ONDO}

Université Omar Bongo de Libreville 